\title{
Research progress of phthalates' endocrine disrupting properties on humans and their usage in the consumer products
}

\author{
Jiang Xian-juan ${ }^{1,}$, , Miao Gui-Dong ${ }^{2, ~ b}$, Wang li-Hong ${ }^{1, c}$, \\ Liu Sheng ${ }^{1, d *}$, Zhang guo-yi ${ }^{2, e}$
}

('Zibo Entry-exit Inspection and Quarantine Bureau of The People's Republic of China, Shandong, Zibo, 255031,china; ${ }^{2}$ College of Biology and Chemistry, Xingyi Normal University for

Nationalities, Xingyi, 562400, China;

aemail: jiangxianjuan@126.com, bemail: guidongmiao@126.com, cemail: 3159573@163.com, ${ }^{d *}$ corresponding author email: sl8181@163.com, eemail: 66884815@qq.com)

Keywords: endocrine disrupting chemicals; phthalates; phthalic acid esters; consumer products

\begin{abstract}
Phthalates, which were widely used as plasticizer and additives in various consumer products, were found to be associated with reproductive disorders in both men and women through their interaction with endocrine glands. These synthetic compounds, which could interfere or disrupt the normal synthesis, secretion, transportation, binding and metabolism of natural hormones were called endocrine disrupting chemicals(EDCs). The EDCs in consumer products might migrate from their source products and creating the potential for exposure. The phthalates were the major occurring EDCs in the consumer products, this paper reviewed their main sources, measured concentration in the consumer products and their endocrine disrupting properties in humans health.
\end{abstract}

\section{Introduction}

Few chemicals on the market today had undergone as much scientific scrutiny as phthalates considering their widely usages as plasticizer and additives in consumer products as well as their endocrine disrupting properties on humans. Phthalates or phthalic acid esters (PAEs) are a class of compounds widely used as plasticizers to provide mechanical strength and flexibility to the resins [1] and they are ingredients of plastics, paints, adhesives, toys, house-building materials, solubilizers of cosmetic products, defoaming agents, PVC pipes, food packing materials, medical devices, photography films, textile fabrics, pesticide carriers, lubricating oils and are used in aerospace technology[2]. In addition, some of PAEs also have endocrine disrupting properties[3] and considered one of the most major occurring endocrine disrupting chemicals (EDCs) . EDCs are exogenous agents that interfere or disrupt the normal synthesis, secretion, transportation, binding and metabolism of natural hormones; eventually dysregulating homeostatic mecha-nisms, reproduction and development[4]. PAEs were not chemically but only non-covalently bound to their polymeric matrix [5-7], so they frequently leach from these items into environmental and their dispersion from matrix can occur at all stages of their use and the exposure to PAEs is almost inevitable[8].

Over the past few decades, large numbers of studies have shown that phthalates were associated with reproductive disorders in both men and women through their interaction with endocrine glands [9, 10], and a variety of diseases stem from their disruption or interference with the endocrine system[11-13] . Nine phthalates were already included in the REACH Candidate List as Substance of Very High Concern (SVHC) based their endocrine disrupting properties or toxic for reproduction properties [14]. In addition, the U.S. Consumer Product Safety Commission (CPSC) also issued a rule prohibiting children's toys and child care articles containing more than 0.1 percent of certain eight phthalates for their toxicities[15].

However, there were dozens of different types phthalates and each have respective structures, properties, toxicities, different applications in consumer products and consequently discriminatory exposure opportunities and differential hazard were available. Aimed at revealing the potential impact of different substandard consumer goods on humans health, the endocrine disrupting properties of PAEs and their potential exposure in consumer goods were studied. 


\section{General applications of PAEs in consumer goods}

The usages of various PAEs depend in part on their molecular weight (MW) and length of their alkyl chains[3,16]. PAEs with higher MW and longer longer alkyl chains such as di-(2-ethylhexyl) phthalate (DEHP), di-isononyl phthalate (DINP), and di-isodecyl phthalate (DIDP) are used as plasticizers in the polymer industry to improve flexibility, workability, and general handling properties for use in construction material, clothing and furnishings, and $80 \%$ of PAEs are used for this purpose.

PAEs with relatively lower MW and shorter alkyl chains such as diethyl phthalate (DEP), dimethyl phthalate (DMP) and dibutyl phthalate (DBP) tend to be used as solvents in perfumes, adhesives, waxes, inks, pharmaceutical products, insecticide materials, and cosmetics[3,16]. DMP and DEP allow perfume fragrances to evaporate more slowly, lengthening the duration of the scent, and a small amount of DBP gives nail polish a chip-resistant property. Single applications may also use mixtures of phthalates.

In 2017, 171 batchs of consumer goods(including 167 toys, 4 childcare articles and children's equipment)were alerted by the Rapid Alert System for dangerous non-food products (RAPEX)[17] because the products contained bis(2-ethylhexyl) phthalate (DEHP) ( measured values within the intervals of $0.24 \%-47 \%$ by weight). Among them, 42 batch consumer goods contained di-butyl phthalate (DBP) (measured values within the intervals of $0.15 \%-27.94 \%$ by weight), 26 batch consumer goods contained di-isononyl phthalate (DINP) (measured values within the intervals of $0.155 \%-34 \%$ by weight) and 2 toys contained di-isodecylphthalate (DIDP)(measured values within the intervals of $0.1 \%-2.3 \%$ by weight). According to the REACH Regulation the phthalates DEHP, DBP and BBP are prohibited in all toys and childcare articles while the phthalates DINP and DIDP are prohibited in toys and childcare articles that can be placed in the mouth by children. The alerted products were recalled from end users or withdrawed from the market.

\section{The endocrine disrupting properties of PAEs}

\subsection{Estrogens disruptors}

The structures of PAEs are similar with those of endogenous estrogens, so they could affect human growth and reproduction by interfering with normal hormone levels in human body[18]. Moreover, PAEs are a group of EDCs that target the ovary to adversely affect the two essential processes of folliculogenesis and steroido-genesis[19]. PAEs have been shown to alter ovarian and oocyte development, target specific follicle types, alter progression of follicular development, and dis-rupt the functionality of follicles and corpora lutea[13]. Some studies highlighted that the developmental toxicity of BBP and DBP and the estrogenic endocrine disrupting activity of BBP, DBP, DEHP, and DINP on intact organisms[20,21]. Some PAEs (DEHP, DBP, BBP) and their metabolites are estrogenic and exhibit adverse reproductive effects[22]. It wasfound that BBP and DBP activated ER transactivity, whereas DEHP antagonized the $17 \beta$-estradiol induced ER function[23].

Exposure to PAEs in women was associated with pre-mature thelarche $\left(96.5 \pm 134.0 \mathrm{ng} \mathrm{\textrm {L } ^ { - 1 }}\right)$, pregnancy loss (MEHP, $377.6 \mathrm{ng} \mathrm{mL}^{-1}$; MBP, $255.1 \mathrm{ng} \mathrm{mL}^{-1}$ ) and other disorders such as smaller pre-ovulatory folli-cles, anovulation or delayed ovulation, longer estrous cycle, decreased synthesis of estradiol, decreased serum progesterone and increased serum follicle-stimulating hormone (FSH) [24,25]. Phthalic acid esters (PAEs) can elicit cellular estrogenic responses and suppress calcium signaling [26-28]. This is concerning for public health because PAEs were used extensively in a wide variety of commonly used items, such as perfumes, nail polish, whitening products and other cosmetics, resulting in ubiquitous human exposure.

\subsection{Androgen disruptors}

PAEs were shown to produce anti-androgenic effects by suppressing testosterone and oestrogen production. At very high phthalate levels, reproductive abnormalities were reported in rodent females which included increased uterine and ovarian weights, and delayed onset of puberty[29]. 
Moreover, it was found that PAEs, dibutyl phthalate(DBP) and diethyl hexyl phthalate(DEHP) that disturb androgen biosynthesis [30, 31]. Phthalates in semen $(0.08-1.32 \mathrm{mg} / \mathrm{L})$ among men are related to de-clined semen quality and infertility [32]. Phthalate monoesters (monoethyl hexyl phthalate, MEHP and monobutyl phthal-ate, MBP) in maternal urine excretion (24.9 and $78.4 \mathrm{ng} \mathrm{L}-1$ ) and exposure of fetus to phthalates in amniotic fluid $(22.8$ and $85.2 \mathrm{ng} \mathrm{mL}-1)$ significantly induced anti-androgenic in male infants characterized by short anogenital distances [33]. Di(2-ethylhexyl)phthalate (DEHP) could disrupt the physical association between Sertoli and germ cells, resulting in premature loss of germ cells from the seminiferous epithelium [34,35]

\subsection{Thyroid hormone disruptors}

Numerous chemicals have been shown to interfere with thyroid function in experimental studies but studies of the thyroid-disrupting effects of phthalates and their monoester metabolites are scarce. In rats, di-n-butyl phthalate (DBP) decreased T3 and T4 in rats in a dose-dependent manner [36], and several studies have shown histopathological changes in the thyroid after exposure to phthalates [37,38]. In vitro studies indicated the antagonistic properties of DBP and DEHP to the thyroid system $[39,40]$. MandanaGhisari etc. [23]found that the mixture of six plasticizers including benzyl butyl phthalate (BBP), dibutyl phthalate (DBP), dioctyl phthalate (DOP), diisodecyl phthalate (DIDP), diisononyl phthalate (DINP), di(2-ethylhexyl) phthalate (DEHP) was evaluated for its thyroid hormone (TH)-like activities by the effect on the TH-dependent rat pituitary GH3 cell proliferation (T-screen). Boas $\mathrm{M}$, et al.found that negative associations between urinary phthalate concentrations and thyroid hormones, IGF-I, and growth in children,this study may suggest causative negative roles of phthalate exposures for child health[11]. For certain groups, such as hospitalized neonates and premature babies, exposure to PAEs may be massive. In these patients, changes in thyroid hormone levels as a result of exposure to phthalates may be transient, but could nonetheless have permanent effects on the development of the central nervous system, if changes occur in a developmentally critical phase[11,23,39].

\section{Conclusions}

Some PAEs are endocrine disrupting chemicals and they can alter one or more functions of the endocrine system and consequently cause adverse health effects in an intact organism. A variety of diseases was caused that stem from their disruption or interference with the endocrine system. Their environmental behavior has attracted considerable attention due to their potential impact on ecosystem functioning and on public health. The use of some PAEs has been restricted in the European Union(EU) since 1999 and in the United States since 2008[41,42].

\section{Acknowledgements}

This work was financially supported by Science and Technology Project of Administration of Quality Supervision, Inspection and Quarantine (No.2016IK191), Guizhou Natural Science Foundation ([2016]7037).

\section{REFERENCES}

[1] C. D. Cartwright, S. A. Owen, I. P. Thompson, R. G. Burns: FEMS Microbiol vol.186(2000), p. 27.

[2] V. Kumar1, S. S. Maitra1: Biotech vol.6 (2016), p. 200.

[3] S. Net, R. Sempere, A. Delmont, A. Paluselli, B. Ouddane: Environ. Sci. Technol vol.4(2015), p. 4019.

[4] J. Annamalai, V. Namasivayam: Environment International vol.76 (2015), p.78. 
[5] A. Berge, M. Cladiere, J. Gasperi, A. Coursimault, B. Tassin, R. Moilleron: Environ Sci Pollut Res Int vol. 20 (2013), p. 8057.

[6] Y. Hongjun , X. Wenjun ,L. Qing ,L. Jingtao, Y. Hongwen, L. Zhaohua: Environ Monit Assess vol.185 (2013), p. 8489.

[7] B. Martine , T. Marie-Jeanne, D. Cendrine , A. Fabrice , C. Marc: Bull Environ Contam Toxicol vol.90 (2013), p. 91.

[8] S. Jobling, T. Reynolds, R. White, M. G. Parker, J. P. Sumpter: Environ Health Perspect vol. 103(1995), p. 582.

[9] Y. H. Zhang, L. X. Zheng, B. H. Chen: Biomed. Environ. Sci. vol.19 (2006), 205.

[10]S. Takeuchi , M. Iida , S. Kobayashi, K. Jin , T. Matsuda , H. Kojima: Toxicology vol. 210 (2005), p. 223.

[11]M. Boas , H. Frederiksen , U. Feldt-Rasmussen , N. E. Skakkebaek , L. Hegedus , L. Hilsted, et al: Environ Health Perspect vol. 118(2010), p.1458.

[12]C. A. Harris, P. Henttu , M. G. Parker, J. P. Sumpter : Environ Health Perspect 105(1997), p. 802.

[13]P. R. Hannon, J. A. Flaws: Frontiers in Endocrinology vol. 6(2015 ), p. 8.

[14]Information on https://echa.europa.eu.

[15]Information on https://www.cpsc.gov.

[16]T. Schettler: International journal of andrology vol.29 (2006), p. 134.

[17]Information on https://ec.europa.eu.

[18]S. Jobling, T. Reynolds, R. White: Environ. Health vol.103 (1995), p. 582.

[19]P. R. Hannon, JA Flaws, The Effects of Phthalates on the Ovary,Frontiers in Endocrinology , 2015, $6: 8$

[20]X.Chen, S. Xu, T. Tan, S. T. Lee, S. H. Cheng, F. W. F. Lee, S. J. L. Xu, K. C.Ho: Int. J. Environ. Res. Public Health vol. 11(2014), p. 3156.

[21] J. Oehlmann, U. Schulte-Oehlmann, W. Kloas, O. Jagnytsch, Lutz etal. Philos. Trans. R. Soc., B, vol 364(2009), p. 2047.

[22]N. Scholz, Chemosphere vol. 53(2003), p.921.

[23]M. Ghisari, E. C. Bonefeld-Jorgensen, Toxicology Letters, vol.189 (2009), p. 67.

[24]Y. Y. Chou, P. C. Huang, C. C. Lee, J. Pediatr, Endocrinol. Metab,vol. 22(2009), p. 69.

[25]G.Toft, B. A. G. Jonsson, C. H. Lindh, T. K. Jensen, N. H. Hjollund, A.Vested, J. P. Bonde, Environ. Health Perspect. vol. 120 (2012), p.458.

[26]N. P. Moore. Reprod Toxicol vol.14 (2000), p.183.

[27]L. Kaun-Yu , T. Fu-Wei , W. Chia-Jung , L. Pei-Shan: Toxicology vol. 200(2004), p. 113.

[28]T. Lovekamp-Swan , B. J. Davis: Environ Health Perspect vol. 111(2003), p. 139.

[29]R. Moral , R. Wang , I. H. Russo , C. A. Lamartiniere , J. Pereira, et al: J Endocrin vol. 196 (2008), p. 101.

[30]E. Mylchreest, et al . Reproductive Toxicology, 16(2002), p. 19.

[31]J. S. Fisher et al: Human Reproduction,vol. 18 (2003), p. 1383.

[32]Y. H. Zhang, L. X. Zheng, B. H. Chen, Biomed. Environ. Sci. vol. 19(2006), p. 205. 
[33]P. C. Huang, P. L. Kuo, Y. Y. Chou, S. J. Lin, C. C. Lee: Environ. Int. vol. 35(2009), p. 14.

[34]P. Erkekoglu , N. D. Zeybek, B. Giray, E. Asan, F. Hincal: Archives of Environmental Contamination and Toxicology vol. 62 (2012), p. 539.

[35]J. S. Fisher: Reproduction vol. 127 (2004), p. 305.

[36] J. C. O’Connor, S. R. Frame, G. S. Ladics: Toxicological Sciences, vol. 69 (2002), p. 92.

[37]J. A. Howarth et al. Toxicology Letters, vol. 121(2001), p. 35.

[38]R. Poon, et al: Food and Chemical Toxicology, vol. 35 (1997), p. 225.

[39] S. Sugiyama, et al: Toxicological Sciences, vol. 88(2005), p. 367.

[40]H. Shen, et al: Chemosphere, vol. 62 (2006), p. 390.

[41] Information on http://europa.eu.

[42]Information on http://www.gpo.gov. 